\title{
Medial septal nucleus and delayed alternation in rats
}

\author{
GARTH J. THOMAS, GILBERTO N. O. BRITO, and DANIEL P. STEIN \\ University of Rochester, Rochester, New York 14642
}

\begin{abstract}
The effects of small electrolytic lesions in the medial septal region on retention of a spatiotemporal task (contingently reinforced T-maze alternation) were determined in Long-Evans rats. With brief intertrial intervals (massed trials), control rats were only slightly (if at all) affected by the 17-day vacation from testing during the period of postoperative recovery.The experimental rats with medial septal lesions, on the other hand, dropped to chance levels of alternation and then recovered to a statistically significant but slight amount over nine sessions of postoperative testing. With long intertrial intervals produced by running the animals in squads of four, control rats dropped to chance levels of alternation and then recovered somewhat over seven additional sessions of testing. Animals with septal lesions tended to perseverate and showed no evidence of recovery. Results were interpreted to indicate that a major hippocampal afferent system (dorsal fornix) plays a crucial role in mediating the spatiotemporal construction and use by the brain of the "cognitive maps" necessary for successful T-maze alternation.
\end{abstract}

The purpose of this study was to evaluate the effect of medial septal lesions on contingently reinforced T-maze alternation. Previous research (Thomas, 1978, 1979; Thomas \& Brito, 1980) had shown that small lesions that ablated approximately the dorsal and posterior one-quarter of the septum had a marked and deleterious effect, which was temporary with regard to an easy problem (brief ITI) on contingently reinforced $\mathrm{T}$-maze alternation, but was permanent with regard to spontaneous alternation. We speculated that the crucial structure was one of the major efferent fiber systems of the hippocampus, the precommissural fornix. The small septal lesions described in the present study have a common focus in the medial septal nucleus, which represents the principal source of one of the major afferent fiber systems to the hippocampus, the dorsal fornix. Consistent with this notion is Winson's (1978) study and an earlier report of Donovick (1968) that lesions in the medial septum that abolished hippocampal theta rhythm also impaired spatial behavior.

The present study was also intended to test the notion that the "recovery of function" that had been previously reported in T-maze alternation following posterodorsal septal lesions depended on the use of other (nonseptal) hypothetical brain circuits. It had been speculated (see Thomas \& Brito, 1980) that projections from medial frontal cortex to entorhinal

The authors are indebted to Jean A. Conlon for assistance with the histological materials and to Louise Behrens for assistance in preparing the manuscript. The study was partially supported by a fellowship (No. PRA-59768) from the Organization of American States to G.N.O.B. For reprints, write to Garth J. Thomas, Center for Brain Research, Box 605, University of Rochester Medical Center, Rochester, New York 14642. cortex via the cingulum bundles (e.g., Beckstead, 1979) mediate the recovery. As indicated below, that hypothesis was not evaluated definitively because the cingulum lesions were not sufficiently successful, and the recovery of alternation after the lesions in the medial septal region, although statistically significant, was not very marked.

The reason for our interest in contingently reinforced T-maze alternation as a behavioral endpoint is our opinion that it represents a highly reliable and easily measurable behavior that comprises a relatively pure instance of a "cognition." As we have pointed out before (Thomas \& Brito, 1980), the idea is old that successful T-maze alternation does not represent a discrimination based on sensory input at the time of choice, but rather a discrimination of a cognition (knowledge) of spatial and temporal aspects of environmental events, that is, which arm of the Tmaze was reinforced last. The logical necessity for the concept of cognition was clearly stated a long time ago by Hunter (1913). As shown in a recent book by O'Keefe and Nadel (1978), there is convincing evidence that the hippocampal formation plays a central role in mediating this spatial aspect of cognitive behavior, at least in mammals. It seems likely that specialized brain circuits that can mediate spatial cognitions ("cognitive maps") will be found in the brains of all animals whose ancestors evolved the necessary behavioral capabilities to forage and locomote effectively with regard to places that are spatially disparate in the environment. As behavioral translocation in space is not instantaneous, nervous systems will have had to evolve a type of "memory" for successful space-time integration of behavior. The current notion of "working memory" (e.g., 
Honig, 1978; also Olton, Becker, \& Handelmann, 1979) reflects one facet of our grappling for understanding of the temporal dimension of behavioral space-time integration.

It has been known for a long time that septal lesions have a deleterious effect on spontaneous Tmaze alternation (e.g., Clody \& Carlton, 1969; Douglas \& Raphaelson, 1966). Contingently reinforced T-maze alternation is also deleteriously affected by small posterodorsal septal lesions (Thomas, 1978, 1979; Thomas \& Brito, 1980). These lesions slightly involved the medial septal nuclei, but their major characteristic was severe disruption of connections between the hippocampus and lateral septal nuclei (precommissural fornix). The present study's major contribution lies in the discovery that small lesions in the medial septal nuclei, the principal origin of the dorsal fornix (e.g., Swanson \& Cowan, 1979), also disrupt contingently reinforced $\mathrm{T}$-maze alternation.

\section{METHOD}

\section{Subjects}

Two shipments of young adult male Long-Evans rats from Blue Spruce Farms (Altamont, New York) were used as subjects. The first shipment weighed a median of $249.5 \mathrm{~g}$ (range, 244$256 \mathrm{~g}$ ). The rats in the second shipment weighed a median of $233 \mathrm{~g}$ (range, 219-251 g). The first shipment provided three rats in each of three experimental groups and one control rat. The second shipment provided three rats for each experimental group, five controls, and two extra rats. One of the extras was substituted as an operated control to replace a loss due to anesthetic death in surgery, and one rat was not used. The experiment was conducted as two replications, about 3 months apart, with 10 rats in the first experiment and 14 rats in the second. As all experimental treatments were represented in each replication and no behavioral differences attributable to shipments were observed, the data are reported as one experiment with 24 rats. The rats were maintained on a reversed light-dark cycle of 12-12 h and were tested in the early afternoon.

\section{Apparatus}

All behavioral testing was conducted in a T-maze made of pine and painted gray. The floor of the maze was made of unpainted Masonite, and the ceiling was made of wire mesh. The startbox (SB; $36 \times 10 \times 14 \mathrm{~cm}$ high) opened directly into a choice area $(15 \times 12 \mathrm{~cm})$ through a guillotine door. Two guillotine doors on each side of the choice area opened into identical goalboxes (GBs) $180 \mathrm{deg}$ apart, and each was $38 \times 10 \times 14 \mathrm{~cm}$ high. Small dishes of wet mash could be pushed into the GBs at the distal ends through slots in the side walls. The rats were observed through a tilted mirror suspended over the maze. The maze was kept clear of detritus, but it was not washed out between trials. Since so little is known about informational content of olfactory input (as opposed to sensitivity) for animals, it seemed more appropriate to confound the cue value of olfactory stimuli by some procedural controls rather than by attempts to eliminate olfactory cues (problematic) by washing the apparatus (see Squire, 1969).

\section{Behavioral Procedures}

Food motivation. After a week on ad-lib food and water, all rats were presumed to have adjusted to living in isolation in laboratory cages-at least, they were all gaining weight. The rats were then put on a reduced-food regimen consisting of a feeding of wet mash once a day immediately after behavioral testing.
Supplementary food was given to keep the rats about $85 \%$ of their ad-lib weights. The rats remained on this reduced-food regimen throughout the experiment, except for 8 days postoperatively when they received ad-lib food. After that, the $85 \%$ of ad-lib weight was recalculated. Water was always available in the home cages.

Adaptation. Before alternation training, the rats received 5 days of adaptation to the T-maze. For each rat, adaptation consisted of being placed for $1 \mathrm{~min}$ in each part of the maze (one SB and two GBs) with a dish of wet mash. Time spent eating was recorded with a stopwatch. The rats received adaptation sessions in irregular order. By the 5th day, all rats were eating steadily, and their exploratory tendency and inhibitory neophobia had largely habituated. It is our belief that, in order for alternation behavior to emerge quickly and strongly, animals must be well adapted to the test apparatus; that is, their exploratory and neophobic tendencies must be minimal.

Alternation. Preoperatively, all rats received nine sessions of training in the $T$-maze at the rate of one session per day. On the first trial of each session, entry into either GB was rewarded by access to wet mash for about $10-15 \mathrm{sec}$. For the next 12 trials, reinforcement (dish of wet mash) was pushed into the GB only if the rat had alternated, that is, if the rat went to the opposite GB from the one in which it last received reinforcement. Thus, the rats were given 12 opportunities in each session to alternate. They were run under conditions of contingent reinforcement for alternating (the "rerun correction procedure"). The intertrial intervals (ITIs), that is, the time it took to remove the rat from the GB and replace it in the SB (circa 8-10 sec), were as brief as practicable.

After postoperative recovery (see below), the rats received another nine sessions of alternation testing under the same conditions as described above, and, after the nine postoperative sessions with brief ITIs, the rats received seven identical sessions with long ITIs. The long ITIs were accomplished by running the rats in squads of four. This produced a median ITI of $104 \mathrm{sec}$ (interquartile range, 99 to $109 \mathrm{sec}$ ).

\section{Surgery}

When the rats had completed nine preoperative sessions of alternation training, they were divided into four groups of six rats each, matched in terms of total alternation score. Six rats comprised the SEP group. They each received electrolytic lesions through a .25 -mm-diam dental arch-wire electrode insulated with Epoxylite except for a bare-metal tip that had been ground to a blunt cone. Lesions were produced by passage of a $1.0-\mathrm{mA}$ anodal current for $15 \mathrm{sec}$ at a stereotaxically determined site in the midline, $6.1 \mathrm{~mm}$ below the skull surface and at an AP of $1 \mathrm{~mm}$ anterior to bregma; the skull was level (bregma and lambda at the same stereotaxic vertical).

A second group of six rats received identical surgical procedures to produce septal lesions; in addition, they received knife-cut lesions aimed at severing the cingulum bundle on each side at an AP level 1-2 mm behind bregma (SEP + CING). Oval holes, with the long axes perpendicular to (and on either side of) the midline, were bored in the skull with a dental burr. The medial ends of the skull holes were within a fraction of a millimeter of the midline. A knife, made from the broken-off tip of a \#11 scapel blade, extended $3.4 \mathrm{~mm}$ beyond the tips of a large hemostat that formed the knife handle. The knife was inserted vertically into the brain for the full depth of the blade $(3.4 \mathrm{~mm})$ as close to the midline as possible and then moved laterally. This procedure was done on both sides.

A third group of six rats received only lesions aimed at severing the cingulum bundles, as described above (CING).

The fourth group of six rats comprised an operated control group (CONT). They underwent identical surgical procedures: $1.6 \mathrm{mg}$ atropine $15 \mathrm{~min}$ prior to anesthesia, anesthesia induced 
by an ip injection of Chloropent $(.3 \mathrm{ml} / 100 \mathrm{~g}$ body weignt), fastening in the stereotaxic head holder, slitting the skin, boring a midline hole in the skull $(.75 \mathrm{~mm}$ diam), and sewing up the skin with interrupted silk sutures.

A comment should be made about inserting the electrode in the midline. The small drill for perforating the skull extended for only about $1.0 \mathrm{~mm}$ beyond the jaws of the jewelers' pin vise that held it. This drill produced a hole in the skull when turned slowly by hand, but, apparently because of its short length, it did not tear the sagittal sinus. In any event, boring the skull hole in no case resulted in sinus bleeding. When the electrode was inserted through the hole for producing a septal lesion, severe sinus hemorrhage occurred with only one rat. The bleeding stopped in about $1 \mathrm{~min}$. It is our hunch that the blunt, cone-shaped tip of the electrode, in most cases, pushed aside the sinus and did not directly puncture it. No evidence of intraskull bleeding was observed (either epi- or subdural) in any of the brains, and the one rat that did show sinus bleeding was behaviorally indistinguishable from other rats in its group.

Following surgery, the rats received 17 days of postoperative recovery. For 8 days, the rats were on ad-lib food (Purina rat pellets). For the last 9 days of postoperative recovery, the rats were put back on the reduced-food regimen of about $7 \mathrm{~g}$ of dry food (given as wet mash) until their weights were approximately $85 \%$ of their new ad-lib weights; they were kept at this $85 \%$ level by small supplements of food given to them at the regular feeding time right after they had been tested in the T-maze. In no case did the small septal lesions induce the state of hyperexcitability of the "septal syndrome" (Thomas, Moore, Harvey, \& Hunt, 1959).

\section{Histology}

At the termination of behavioral testing, the rats were killed with an overdose of Nembutal and perfused through the heart with buffered $10 \%$ Formalin. The brains were removed from the skulls and, after further hardening in $10 \%$ Formalin, were embedded in celloidin (Randolph Products), sectioned at $48 \mu$, and every fourth section through the lesions was mounted and stained with cresyl violet. The cell stain does not, of course, directly visualize fiber bundles, so the lesions aimed at cutting the cingulum bundle were evaluated in terms of the location of the lesions in relation to the area occupied by the cingulum bundle in fiber-stained material. The cingulum-bundle lesions were not very successful (see below), but a photomicrograph illustrates the nature of the lesions (see Figure 1).

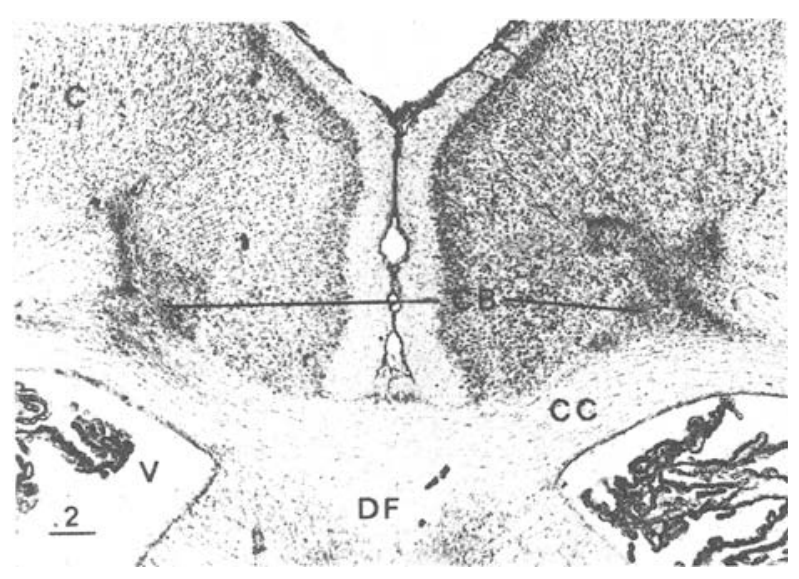

Figure 1. Photomicrograph showing knife-cut lesions that achieved a nearly complete bilateral transection of the cingulum bundles. Cresyl-violet stain. Abbreviations: C, cortex; CB, cingulum bundles; $\mathrm{CC}$, corpus callosum; DF, dorsal fornix; V, ventricle. Calibration $=.2 \mathrm{~mm}$.
The septal lesions are very complex to illustrate. There is substantial distortion of remaining brain tissue, presumably due to collapse of the cavity in the center of the lesions, with consequent "inflow" of normal brain tissue and thus apparent shrinkage of the lesions. This change is also accompanied by an enlargement of the ventricles, especially the vertical slits of the lateral ventricle on either side of the septal area. A reconstruction of the lesion in terms of remaining normal tissue projected onto brain sections from a normal brain is believed to be more useful for illustrative purposes than photomicrographs.

\section{RESULTS}

\section{Anatomical}

Results from the groups receiving lesions aimed at bilaterally transecting the cingulum bundles can be dealt with quickly. The knife-cut technique was not effective. In the CING group, only one rat received a nearly complete bilateral cut, as illustrated in Figure 1. All the rest received only partial cuts. In the SEP + CING group, there were two animals with complete cuts. Apparently, the knife just pushed aside the fiber bundles instead of cutting them. As might be expected from the results of Greene and Stauff (1974), the alternation behavior of one animal with complete bilateral transection of the cingulum bundles was in no way distinguishable from the other rats in its group or from the control rats with no brain damage.

It had originally been hypothesized that cutting the cingulum bundles would prevent the recovery of function in alternation seen after septal lesions. The hypothesis was not tested definitively in this experiment for two reasons. In the first place, although some "recovery of function" occurred (see Figures 3 and 4) after these septal lesions, it was not nearly as striking as that observed previously after posterodorsal septal lesions (Thomas \& Brito, 1980). In the second place, the "recovery of function" in the only two animals with complete bilateral transection of the cingulum and septal lesion was not conspicuously different from that shown by other animals with septal lesions.

Since the main purpose of this experiment was an evaluation of the more anterior septal lesions on contingently reinforced T-maze alternation, the scores of the CING group were combined with the scores of the CONT group, making a new control group of 12 rats, 6 operated control and 6 lesion control rats $(\mathrm{CO})$. Scores of the SEP + CING group were combined with scores of the SEP group, making a new experimental group of 12 rats with midseptal lesions (EX). From a "mass action" point of view, the lesions in the cingulum were very small and had no detectable effect on performance.

Figure 2 illustrates a frontal and sagittal reconstruction of two septal lesions. Shown in the top lefthand panel is a reconstruction of the lesion on a frontal brain section from a normal rat of the same strain and sex at the AP level of the center of the lesion. On the right panel is a reconstruction of the 


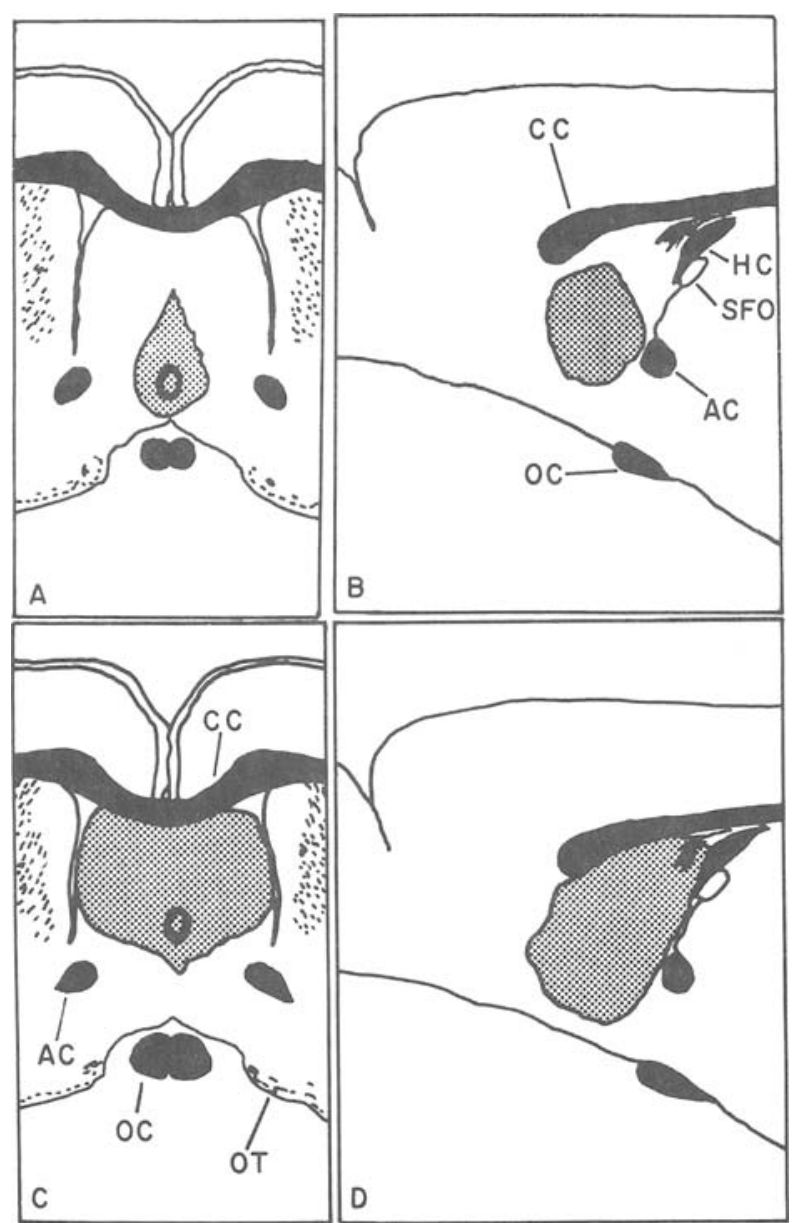

Figure 2. Schematic drawings reconstructed to illustrate the septal lesions. The top two panels ( $A$ and $B$ ) show frontal and sagittal views of the smallest lesion. The bottom two panels $(C$ and $D)$ illustrate the largest lesion. The lesion is indicated by shading. The ovals within the shaded areas indicate the location of the electrode tip. Abbreviations: AC, anterior commissure; $\mathbf{C C}$, corpus callosum; HC, hippocampal commissure; OC, optic chiasma; OT, olfactory tubercle; SFO, subfornical organ.

lesion in a schematic sagittal view. The subcortical structures were traced from a midline section; the neocortex and olfactory bulbs were traced from a section located about $.5 \mathrm{~mm}$ lateral to the midline. The top two panels illustrate the smallest lesion; the bottom two panels show the largest lesion. Microscopic examination of each section showed that the common area of the lesions was very similar to that indicated in Panel A of Figure 2; that is, the lesions were centered in the more ventral region of the medial septal nucleus where it merges with the nucleus of the diagonal band of Broca. However, in some brains, individual lesions extended into the lateral septal nuclei; in other brains, the taenia tecta were obliterated; in no case did the lesions extend across the ventricle and damage the caudate nuclei; in one case, there was more extensive damage to the corpus callosum than the small area of damage produced by penetration of the electrode; and in only one instance was the alternation behavior of a rat.in the group with septal lesions strikingly different from that of the rest of the animals in that group. In all cases but Rat 17, alternation fell to chance levels on the first postoperative test. Rat 17 made $92 \%$ alternations, well within the range of control rats. However, on subsequent sessions, its scores fell to a much lower percentage. Inadvertently, its lesion was made $1.8 \mathrm{~mm}$ too far forward. The result was brain damage that extended anteriorly beyond the genu of the corpus callosum into the ventral prelimbic cortex (small); the lesion ablated all of the anterior septal area. More posteriorly, the lesion became unilateral, taking out much of one lateral septal nucleus and about half of the anterior part of the medial septal nucleus. At its posteriormost end, the lesion again became small and invaded the midline region of the posterior part of the septum. Critical septohippocampal circuits must have been damaged, because, except for the first session, Rat 17 never performed as well as controls, and with a harder problem (the long ITI), it became a consistent perseverator and achieved reinforcement on only $14 \%$ of the trials.

Of central significance is the fact that all rats in the EX group (except Rat 17) behaved similarly, and none of the variations in the lesions were related to unique characteristics of behavior. All rats shared near-complete ablation of the medial septal area.

\section{Behavioral}

Figure 3 shows postoperative curves for median scores of the control animals (CO) and the experi-

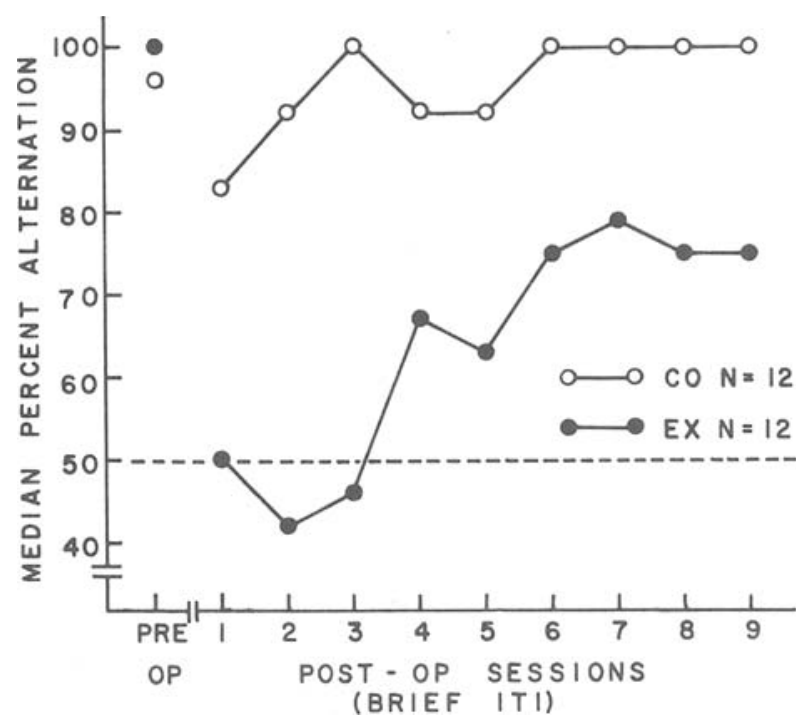

Figure 3. Median percentage alternation as a function of nine daily postoperative sessions with brief ITIs. The two points at the upper left indicate median performance on the last preoperative session. 
mental animals (EX) with septal lesions. The two points in the upper left portion of the graph indicate the level of performance of both groups on the last preoperative trial. As can be seen, the $\mathrm{CO}$ animals are not severely affected by their 17-day vacation from testing during postoperative recovery. Animals with septal lesions were severely affected. During the first three sessions, their medians were close to chance expectancy. Overall, there is an upward trend and it is significant $(\mathrm{p}<.05$, Wilcoxon test), but clearly the animals never recovered to control levels. Summed across all nine postoperative sessions, the controls (CO) made a median of 103 (out of 108 possible) alternations. The animals with septal lesions (EX) made a median of only 70.5 alternations. The difference (no overlap) is significant at $\mathrm{p}<.002$ (twotailed U test).

Clearly, septal lesions that destroyed most of the medial septal nucleus but spared the posterodorsal part of the septal area were effective in disrupting T-maze alternation. Posterodorsal septal lesions have been reported to cause a similar behavioral effect (Thomas, 1978, 1979; Thomas \& Brito, 1980). The addition of cingulum transection to the septal lesions had no apparent effect, but this finding is not definitive.

Running the animals in squads of four to produce longer ITIs had the expected effect, as has been reported before (Thomas \& Brito, 1980). Figure 4 shows

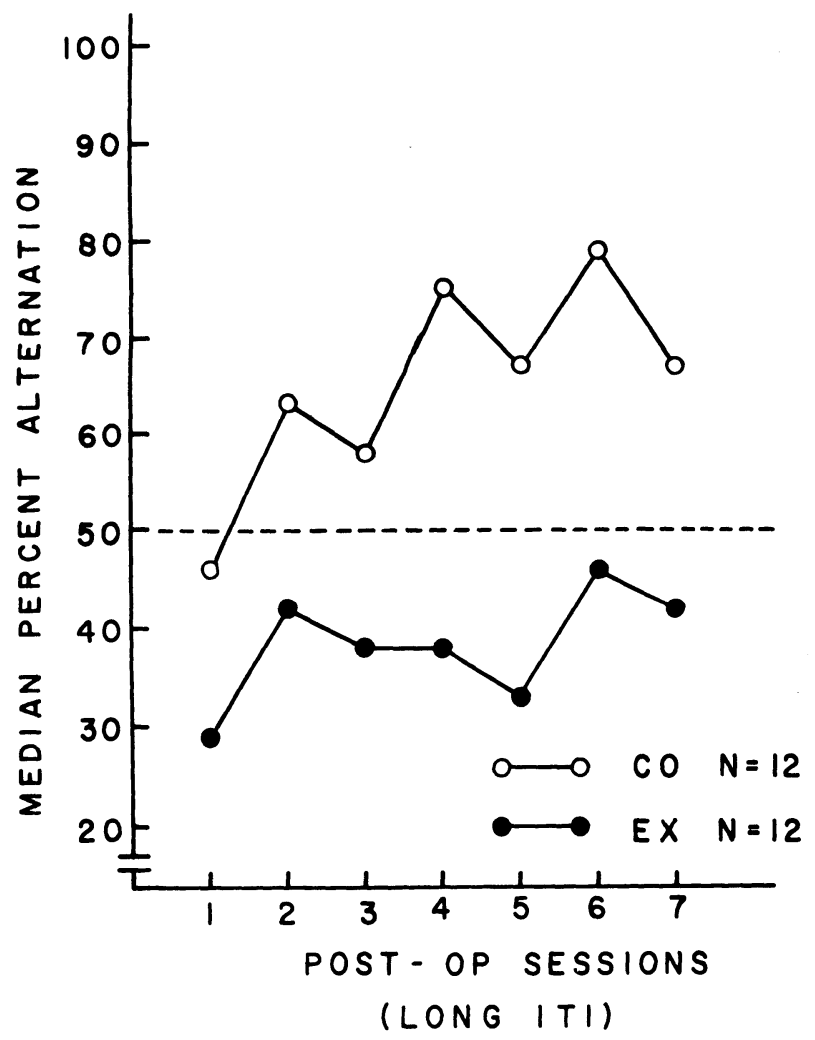

Figure 4. Median percentage alternation as a function of seven daily postoperative sessions with long ITIs (the same animals as in Figure 3). that rats of the control group dropped to a level of alternation performance that was indistinguishable from random choices, and, although they recovered to a statistically significant degree over the seven sessions (Wilcoxon test, first vs. seventh session, $\mathrm{p}<$ $.01)$, the recovery was not striking. They did not do nearly as well on the seventh session with long ITIs as they had done on the ninth session with short ITIs.

Animals with lesions that substantially ablated the medial septal region lost what ability they had recovered during the nine sessions with brief ITIs and were never able to alternate above a chance level. In terms of total alterations across all seven sessions, the EX animals were significantly worse than the $\mathrm{CO}$ animals $(\mathrm{p}<.002$, two-tailed $\mathrm{U}$ test).

\section{DISCUSSION}

The hypothesis that the recovery of function in alternation ability after posterodorsal septal lesions (Thomas \& Brito, 1980) depends on connections from the medial frontal cortex to the entorhinal area via the cingulum bundles (as described by Beckstead, 1979) could not be tested in this experiment because the knife-cut lesions were not successful in enough cases and the degree of recovery of function after the septal lesions was not as marked as expected. Thomas and Brito (1980) speculated that the degree of recovery of function would depend on an interactive effect between the difficulty of the problem (its demand on hypothetical brain circuits subserving spatiotemporal behavior) and the location of the lesions (how much of the hypothetical circuitry the lesions removed). We have no explanation concerning the failure of the control rats to recover as strikingly as was observed earlier (Thomas \& Brito, 1980). However, it should be noted that some conditions in this experiment and in the former one were different, including the ITIs and the T-maze.

It has been known for a long time that septal lesions impaired rats' ability to do spontaneous alternation (e.g., Clody \& Carlton, 1969; Douglas \& Raphaelson, 1966). Previous results from the present series of studies have shown that small lesions that ablated the posterodorsal part of the septum disrupted not only spontaneous alternation (Thomas, 1979) but also contingently reinforced alternation (Thomas, 1978; Thomas \& Brito, 1980). However, with the easy problem (short ITIs), the effect was only temporary with regard to contingently reinforced alternation. It was speculated that the effectiveness of the lesions derived from the fact that the lesions severely damaged a major efferent system of the hippocampus, the precommissural fornix. Similar lesions that abolish theta (and also other, e.g., chemical, septohippocampal influences) interfere with other forms of spatial behavior (Donovick, 1968; Winson, 1978).

The results from the present study indicate that small lesions, having, as a common focus, damage 
to the medial septal nucleus and the dorsal part of the nucleus of the diagonal band, cause a similar and severe loss of contingently reinforced T-maze alternation behavior, as also do posterodorsal septal lesions, but the effect is less temporary (i.e., the recovery of alternation with continued practice is not marked). This observation, in conjunction with studies showing that transection of the whole fornix impairs spatial behavior (e.g., O'Keefe, Nadel, Keightly, \& Kill, 1975), suggests that the crucial aspect of the septal lesions as regards T-maze alternation is the damage to a major afferent system to the hippocampus, the dorsal fornix (Swanson \& Cowan, 1979).

\section{REFERENCES}

Beckstead, R. M. An autoradiographic examination of corticocortical projections of the mediodorsal-projection (prefrontal) cortex in the rat. Journal of Comparative Neurology, 1979, $184,43-62$.

Clody, D. E., \& Carlton, P. L. Behavioral effects of lesions of the medial septum of rats. Journal of Comparative and Physiological Psychology, 1969, 67, 344-351.

Donovick, P. J. Effects of localized septal lesions on hippocampal EEG activity and behavior in rats. Journal of Comparative and Physiological Psychology, 1968, 66, 569-578.

Douglas, R. J., \& Raphaelson, A. C. Spontaneous alternation and septal lesions. Journal of Comparative and Physiological Psychology, 1966, 62, 320-322.

Greene, E., \& Stauff, C. Behavioral role of hippocampal connections. Experimental Neurology, 1974, 45, 141-160.

HonIG, W. K. Studies in working memory in the pigeon. In S. H. Hulse, H. Fowler, \& W. K. Honig (Eds.), Cognitive processes in animal behavior. Hillsdale, N.J: Erlbaum, 1978.
Hunte R, W. S. The delayed reaction in animals and children. Behavior Monographs, 1913, 2, 1-86.

O'KEEFE, J., \& NADEL, L. The hippocampus as a cognitive map. Oxford: Clarendon Press, 1978.

O’Keefe, J., Nadel, L., Keightley, S., \& Kill, D. Fornix lesions selectively abolish place learning in the rat. Experimental Neurology, 1975, 48, 152-166.

Olton, D. S., Becker, J. T., \& HandelmanN, G. E. Hippocampus, space, and memory. The Behavioral and Brain Sciences, 1979, 2, 313-365.

Squire, L. R. Effects of pretrial and posttrial administration of cholinergic and anticholinergic drugs on spontaneous alternation. Journal of Comparative and Physiological Psychology, $1969,69,69-75$.

Swanson, L. W., \& Cowan, W. M. The connections of the septal region in the rat. Journal of Comparative Neurology, 1979, 186, 621-655.

Thomas, G. J. Delayed alternation in rats after pre- or postcommissural fornicotomy. Journal of Comparative and Physiological Psychology, 1978, 92, 1128-1136.

Tномаs, G. J. Comparison of effects of small lesions in posterodorsal septum on spontaneous and re-run correction (contingently reinforced) alternation in rats. Journal of Comparative and Physiological Psychology, 1979, 93, 685-694.

Thомas, G. J., \& Brito, G. N. O. Recovery of delayed alternation in rats after lesions in medial frontal cortex and septum. Journal of Comparative and Physiological Psychology, 1980, 94, 808-818.

Thomas, G. J., Moore, R. Y., Harvey, J. A., \& Hunt, H. F. Relations between the behavioral syndrome produced by lesions in the septal region of the forebrain and maze learning of the rat. Journal of Comparative and Physiological Psychology, 1959, 52, 527-532.

Winson, J. Loss of hippocampal theta rhythm results in spatial memory deficit in the rat. Science, 1978, 201, 160-163.

(Received for publication June 12, 1980; revision accepted July 21,1980 .) 